archives-ouvertes

\title{
Effect of observed micropolar motions on wave propagation in deep Earth minerals
}

Rafael Abreu, Christine Thomas, Stéphanie Durand

\section{To cite this version:}

Rafael Abreu, Christine Thomas, Stéphanie Durand. Effect of observed micropolar motions on wave propagation in deep Earth minerals. Physics of the Earth and Planetary Interiors, Elsevier, 2018, 10.1016/j.pepi.2017.04.006 . hal-02944841

\section{HAL Id: hal-02944841 \\ https://hal.archives-ouvertes.fr/hal-02944841}

Submitted on 21 Sep 2020

HAL is a multi-disciplinary open access archive for the deposit and dissemination of scientific research documents, whether they are published or not. The documents may come from teaching and research institutions in France or abroad, or from public or private research centers.
L'archive ouverte pluridisciplinaire HAL, est destinée au dépôt et à la diffusion de documents scientifiques de niveau recherche, publiés ou non, émanant des établissements d'enseignement et de recherche français ou étrangers, des laboratoires publics ou privés. 


\title{
Effect of observed micropolar motions on wave propagation in deep Earth minerals
}

\author{
Rafael Abreu ${ }^{\mathrm{a}, \mathrm{b}, *}$, Christine Thomas ${ }^{\mathrm{a}}$, Stephanie Durand ${ }^{\mathrm{a}}$ \\ a Institut für Geophysik, Westfälische Wilhelms-Universität Münster, Corrensstraße 24, D-48149 Münster, Germany \\ ${ }^{\mathrm{b}}$ Instituto Andaluz de Geofisica, Universidad de Granada, Campus de Cartuja s/n, E-18071 Granada, Spain
}

\section{A R T I C L E I N F O}

\section{Article history:}

Received 7 February 2017

Received in revised form 7 April 2017

Accepted 25 April 2017

Available online $\mathrm{xxxx}$

\section{Keywords:}

Computational mineralogy

Wave propagation

Bridgmanite

$\mathrm{MgSiO}_{3}$ silicate perovskite

Potassium nitrate

\begin{abstract}
A B S T R A C T
We provide a method to compute the Cosserat couple modulus for a bridgmanite $\left(\mathrm{MgSiO}_{3}\right.$ silicate perovskite) solid from frequency gaps observed in Raman experiments. To this aim, we apply micropolar theory which is a generalization of the classical linear elastic theory, where each particle has an intrinsic rotational degree of freedom, called micro-rotation and/or spin, and which depends on the so-called Cosserat couple modulus $\mu_{c}$ that characterizes the micropolar medium. We investigate both wave propagation and dispersion. The wave propagation simulations in both potassium nitrate $\left(\mathrm{KNO}_{3}\right)$ and bridgmanite crystal leads to a faster elastic wave propagation as well as to an independent rotational field of motion, called optic mode, which is smaller in amplitude compared to the conventional rotational field. The dispersion analysis predicts that the optic mode only appears above a cutoff frequency, $\omega_{r}$, which has been observed in Raman experiments done at high pressures and temperatures on bridgmanite crystal. The comparison of the cutoff frequency observed in experiments and the micropolar theory enables us to compute for the first time the temperature and pressure dependency of the Cosserat couple modulus $\mu_{c}$ of bridgmanite. This study thus shows that the micropolar theory can explain particle motions observed in laboratory experiments that were before neglected and that can now be used to constrain the micropolar elastic constants of Earth's mantle like material. This pioneer work aims at encouraging the use of micropolar theory in future works on deep Earth's mantle material by providing Cosserat couple modulus that were not available before.
\end{abstract}

\section{Introduction}

In order to describe various phenomena related to the microscale structures, which cannot be accounted for by classical continuum mechanics, various generalized theories for elastic continua have been developed, namely micro-continuum field theories (Eringen, 1999). Micro-continuum field theories is a family of theories with enriched kinematics, where each different particle inside the elastic medium has intrinsic degrees of freedom, (Mindlin, 1964). They are mainly divided into three different categories: micromorphic, microstretch and micropolar media.

The micropolar medium, also called the Cosserat's medium (Cosserat et al., 1909), is the simplest case: it describes materials with additional rotational degrees of freedom at each point (Eringen and Kafadar, 1976; Nowacki, 1986; Jeong and Neff, 2010; Neff and Jeong, 2009). In other words, each particle of the

\footnotetext{
* Corresponding author at: Institut für Geophysik, Westfälische Wilhelms Universität Münster, Corrensstraße 24, D-48149 Münster, Germany.

E-mail address: abreu@uni-muenster.de (R. Abreu).
}

elastic medium can independently rotate. This rotational degree of freedom is called spin or micro-rotation and will be hereafter denoted $\vartheta$. The theory assumes that this spin field is different from the continuum rotation.

The theory of microstretch media describes elastic materials with rotational and stretching degrees of freedom (Eringen, 1999). In other words, each particle inside the elastic material can independently rotate and possess stretching motions. The main inconvenience with the microstretch theory is the large amount of elastic constants that have to be determined in order to be successfully applied. Indeed, it requires detailed and well calibrated laboratory experiments which until today prevented any realistic application of the microstretch theory.

The theory of micromorphic media was formulated with the purpose to describe features that the Cosserat's (micropolar) and microstretch theories cannot describe (Eringen, 1999). In particular, it includes any kind of micro-distortion in the elastic material. Despite its generality, like microstretch theory, it raises the problem of the big amount of material parameters that are needed for any application of this theory. 
As a consequence, we will focus in this study on the theory of micropolar media. According to Mindlin (1964) there are three main areas where the application of the micropolar theory is justified:

- Zones with large stress gradients, for instance in the vicinity of holes and/or cracks.

- Waves with high frequency (ultrasonic waves).

- Granular materials or bodies with large molecules, such as polymers.

The theory of micropolar media has been applied successfully. For instance it has been used to model the dielectric relaxation process observed in many types of perovskites (e.g. Randall et al., 1990; Krupanidhi, 2003; Peláiz-Barranco et al., 2005; Heywang et al., 2008; Borkar et al., 2014; Smiga and Ukrainian, 2012). Indeed, the polarization mechanism in the material originates from micropolar clusters, their dynamic nature and, from the induced ferroelectric transition in the non-polar micro-regions. More generally, micro-continuum field theories have been applied for modelling microscopic processes (Madeo et al., 2016a,b,c), as well as macroscopic processes (Teisseyre, 1973; Teisseyre et al., 2006; Nagahama and Teisseyre, 2000a,b; Teisseyre, 2008a,b; Teisseyre, 2011; Twiss, 2009; Twiss et al., 1993). The term micro in the name of the theory refers to a reference scale: for instance, it can refer to the rotation of kilometre size blocks in the Earth's crust, which, compared to the total size of the Earth's crust, behaves as a microscopic motion (Twiss, 2009; Twiss et al., 1993).

The presence of micropolar effects in crystalline solids have also been observed through several laboratory experiments like X-ray diffraction, neutron diffraction, specific heat measurements and infra-red and Raman spectroscopy (Hemley et al., 1987; Karki et al., 2000; Durben and Wolf, 1992; Chopelas, 1996; Williams et al., 1987; Shim et al., 2007). During these experiments some micropolar elastic constants for potassium nitrate $\left(\mathrm{KNO}_{3}\right)$, have been determined and compared to lattice models (Pouget et al., 1986a).

Like silicate perovskite minerals found in the Earth's mantle, potassium nitrate, at room temperature, has an orthorhombic crystal structure. In fact, the orthorhombic crystal structure discussed for $\mathrm{KNO}_{3}$ is representative for a large class of crystalline solids like $\mathrm{BaTiO}_{3}, \mathrm{NaNO}_{3}$, all perovskite crystals, aromatic crystals and hydrogen-bonded crystals (Eringen, 1999). The corresponding micropolar moduli for these crystals can be calculated by means of the appropriate lattice parameters, and/or from frequencies of various modes found in the laboratory.

As mentioned before, one of the main drawbacks of using micro-continuum field theories for the description of the elastic wave propagation is the lack of knowledge of the micropolar elastic constants and their physical interpretations. This problem has been addressed in several studies (Eringen, 1999; Pouget et al., 1986a; Madeo et al., 2016a), however, no general agreement is found in the literature.

The use of micro-continuum field theories for the description of realistic physical problems is wide and there are still many open questions. In this study we focus on the description of the elastic wave propagation using the micropolar model at ultrasonic frequencies so that we take into account the local information of the crystallographic medium. We also propose a method to compute the Cosserat couple modulus for deep Earth's mantle-like material using experimental results. This is to our knowledge the first time that such a catalogue of Cosserat couple moduli is provided for application to deep mantle materials.

In the following, we first give a brief introduction to the theory of micropolar media, explaining strain and stress concepts. Second, we present our simulations to model the elastic wave propagation using the micropolar theory in potassium nitrate $\left(\mathrm{KNO}_{3}\right)$ and bridgmanite crystals. Then, we study the dispersion behaviour predicted by the micropolar theory and use the observation of this behaviour in experiments to propose a Cosserat couple modulus values for bridgmanite. Finally, we draw conclusions related to the applicability of the micropolar media for the solution of the elastic wave propagation problem.

\section{Micropolar theory}

As explained before, the distinguishing feature of micropolar theory is the introduction of a material particle rotation that is independent of the classical continuum rotation. As a consequence, in the case of the linear Cosserat model, additional terms appear in the strain $\left(e_{i j}\right)$ and stress $\left(\sigma_{i j}\right)$ tensors as shown in the following expressions

$$
\begin{aligned}
e_{i j} & =\underbrace{\frac{1}{2}\left(\frac{\partial u_{i}}{\partial x_{j}}+\frac{\partial u_{j}}{\partial x_{i}}\right)}_{\text {symmetric part }}+\underbrace{\sum_{k, a, b=1}^{3} \epsilon_{i j k}(\frac{1}{2} \overbrace{\epsilon_{k a b} \frac{\partial u_{a}}{\partial x_{b}}}^{\text {curl u }}-\overbrace{\vartheta_{k}}^{\text {spin }})}_{\text {antisymmetric part }}, \quad i, j \in\{1,2,3\} ; \\
\sigma_{i j} & =\mu\left(\frac{\partial u_{j}}{\partial x_{i}}+\frac{\partial u_{i}}{\partial x_{j}}\right)+2 \mu_{c} \sum_{k, a, b=1}^{3} \epsilon_{i j k}\left(\frac{1}{2} \epsilon_{k a b} \frac{\partial u_{a}}{\partial x_{b}}-\vartheta_{k}\right)+\lambda \delta_{i j} \sum_{k=1}^{3} \frac{\partial u_{k}}{\partial x_{k}}, \quad i, j \in\{1,2,3\},
\end{aligned}
$$

where $u$ is the displacement vector, $\vartheta$ is spin or micro-rotation vector, $\epsilon_{i j k}$ the Levi-Civita symbol and $\delta$ is the Dirac delta function. The parameters $\lambda$ and $\mu$ are the classical Lamé moduli. It is noteworthy that a new elastic constant, $\mu_{c}$, is introduced. It is called the Cosserat couple modulus and it couples the macro and the micro medium. The main difference between the linear elastic and the micropolar models is the presence of the antisymmetric part in the strain tensor $e_{i j}$ (see Eq. (2.1)), which is absent from in the linear elastic model. If $\mu_{c}=0$ we recover the conventional expression for the symmetric stress tensor. The antisymmetric part is given by the difference between the macro rotation- $\frac{1}{2} \operatorname{curl} u$-and spin $\vartheta$. It means that the micropolar model allows the macro rotation to be different from the spin $\vartheta$; if they are the same, we simply recover the conventional linear elastic model. Finally, we can mention that the micropolar theory only influences shear waves (Eringen, 1999), which means that the equation of motion for $P$ waves in a micropolar media is the same as for linear elastic media. For a more detailed introduction to the theory of micropolar media the reader should refer to Eringen (1999).

In this study we consider the $1 \mathrm{D}$ horizontally polarized $\mathrm{S}$ wave $(\mathrm{SH})$ in a micropolar medium governed by the following coupled PDE's

$$
\begin{aligned}
& \frac{\partial^{2} u_{2}}{\partial t^{2}}=\frac{\mu+\mu_{c}}{\rho} \frac{\partial^{2} u_{2}}{\partial x_{1}^{2}}-\frac{2 \mu_{c}}{\rho} \frac{\partial \vartheta_{3}}{\partial x_{1}}, \\
& \frac{\partial^{2} \vartheta_{3}}{\partial t^{2}}=\frac{\mu L_{c}^{2}}{\eta} \frac{\partial^{2} \vartheta_{3}}{\partial x_{1}^{2}}+\frac{2 \mu_{c}}{\eta} \frac{\partial u_{2}}{\partial x_{1}}-\frac{4 \mu_{c}}{\eta} \vartheta_{3} .
\end{aligned}
$$

where $x_{1}$ represents the $x$ direction, $u_{2}$ is the displacement field in the $y$ direction and $\vartheta_{3}$ is the spin field in the $z$ direction. The term $L_{c}$ is the characteristic length of the problem and $\eta$ the microinertia density. The elastic term $\mu L_{c}^{2}$ is a simplification of the original elastic constants required in the micropolar model and it is equivalent to the hypothesis that $\nabla \cdot \vartheta$ and $\nabla \times \vartheta$ do not cause any stress in the medium (Neff and Jeong, 2009; Neff et al., 2010a,b). There exist continua that do not satisfy this restriction such as for instance electromagnetic media, however, it is useful for our purposes. 
A more simplified model is called the reduced dynamic Cosserat model (Grekova et al., 2009), or internal variable model (Forest and Sievert, 2003), obtained when considering the term $\mu L_{c}=0$ in the equations of motion (Eq. 2.2). It is used when no assumption of the characteristic length $L_{c}$ of the problem is made and it shows different wave propagation properties as we will show in the next sections.

Eq. (2.2) reveals the existence of a cutoff frequency $\omega_{r}$, which corresponds to the frequency at which independent spin motions start to appear. It is defined as follows

$\omega_{r}=\sqrt{\frac{4 \mu_{c}}{\eta}}$

The cutoff frequency $\omega_{r}$ can be interpreted also as an eigenfrequency of a rotational oscillator with the inertia density $\eta$, as one of the point-body of the medium, and elastic constant $4 \mu_{c}$. Note that if the critical frequency $\omega_{r}$ and micro-inertia density $\eta$ are known, it is then possible to compute from Eq. (2.3) the Cosserat couple modulus $\mu_{c}$.

The system of Eqs. (2.2) can be solved numerically so that we can analyse the effect of micorpolar motions on the wave propagation. The only requirement is to know the Cosserat couple modulus, $\mu_{c}$, and to consider a characteristic length of the problem, $L_{c}$. If not provided in the literature, the Cosserat couple modulus $\mu_{c}$ can be computed using Eq. (2.3). It, however, still requires a value of the micro-inertia density $\eta$ that can be approximated by the following expression

$\eta \approx \rho L_{c}^{2}$

(for further details see (Eringen, 1999; Pouget et al., 1986a). We are therefore able to perform simulations of wave propagation in a micropolar medium.

\section{Micropolar simulations}

\subsection{In potassium nitrate $\mathrm{KNO}_{3}$ crystals}

Before considering the elastic wave propagation phenomena in perovskite-like structures including micro-rotational fields, we first focus on potassium nitrate $\mathrm{KNO}_{3}$ crystals for which the micropolar elastic constants have been measured (Eringen, 1999). However, they are given for an anisotropic orthorhombic crystal structure that cannot be used directly in Eq. (2.2) since we are using isotropic equations. In order to overcome this problem, we thus compute the equivalent 1-D isotropic constants which provides values of the shear modulus $\mu$ and Cosserat modulus $\mu_{c}$, and allows us to simulate the wave propagation in a micropolar medium and to make comparisons between the micropolar and linear elastic models. Details about how to find the isotropic equivalent medium are given in Appendix $\mathrm{A}$, and the results are given in Table 1 (first row).

We then simulate, using the Finite-Difference method (FDM), the elastic wave propagation through a perfect alignment of potassium nitrate $\mathrm{KNO}_{3}$ crystals. For details regarding the numerical discretization using the FDM see Appendix B. The parameters used to run the simulation are presented in Table 2. In this simulation, we consider a Ricker source time function located in the displacement field only (see Appendix B).

Fig. 1(a-c) shows the obtained displacement (a), macroscopic rotation- $\frac{1}{2}$ curlu-(b) and microscopic rotation (c). Also displayed for comparison, are the results obtained with the conventional linear elastic theory (red curves). We can observe that the amplitude of the seismograms (Fig. 1) are similar but the micropolar model produces faster waves. The measured velocity contrast between the velocity predicted by the micropolar theory and the conventional linear elastic theory is given by $\sqrt{1+\mathrm{r}_{\mu_{c}, \mu}}$ where $\mathrm{r}_{\mu_{c}, \mu}=\frac{\mu_{c}}{\mu}$ is called the Cosserat ratio and it is equal to 0.0035 in the case of potassium nitrate (see Table 1). We can also observe that the micropolar model predicts a spin field $\vartheta$ which is smaller in amplitude and has a different waveform compared to the macrorotational field- $\frac{1}{2}$ curl $u-$.

\subsection{In bridgmanite $\left(\mathrm{MgSiO}_{3}\right)$ crystals}

In this section we want to consider a more realistic case and therefore test the effect of micropolar motions on wave propagation in deep Earth's mantle-like material. Bridgmanite may form up to $93 \%$ of the Earth's lower mantle (Murakami et al., 2012). With the micropolar elastic constants given by Eringen (1999), measured at ambient conditions, and according to PREM model (Dziewonski and Anderson, 1981), one could simulate micropolar elastic wave propagation through a bridgmanite-like material close to the surface of the Earth. However, for a realistic micropolar simulation of elastic wave propagation within the Earth's lower mantle pressure and temperature conditions (between 670 and $2700 \mathrm{~km}$ depth), all the elastic constants have to be computed at the appropriate depth. From he study by Oganov and Ono (2004), we obtain values of the an equivalent isotropic shear modulus $\mu=310.9 \mathrm{GPa}$, shear wave velocity $v_{s}=7636 \mathrm{~m} / \mathrm{s}$ and density $\rho=5331.98 \mathrm{~kg} / \mathrm{m}^{3}$, at pressures of $120 \mathrm{GPa}$ (close to the Earth's core-mantle boundary) for bridgmanite.

In order to perform the numerical simulation we compute the Cosserat couple modulus $\mu_{c}$. To do this we can apply Eq. (2.3) since this cutoff frequency has been measured in laboratory experiments (Hemley et al., 1987; Karki et al., 2000; Durben and Wolf, 1992; Chopelas, 1996; Williams et al., 1987; Shim et al., 2007). Elastic wave propagation in crystal structures is described by two types of modes: the acoustic one and the optic one. In the acoustic type (longitudinal acoustic LA and/or transverse acoustic TA), all the atoms in the unit cell move in phase, this means that the atoms move coherently in the lattice, resulting in the deformation of the lattice (see Fig. 3). In the optic type (longitudinal optic LO and/or transverse optic TO), the atoms move out of phase (see Fig. 3). In micropolar theory, we relate the frequency at which the optic modes are observed in laboratory experiments to the cut-off frequency that appears in the micropolar wave equations (Eq. 2.2). For instance, in several Raman spectroscopy experiments done on bridgmanite, micropolar and microstretch behaviour of the $\mathrm{SiO}_{6}$ octahedron have been observed (Hemley et al., 1987; Karki et al., 2000; Durben and Wolf, 1992; Chopelas, 1996; Williams et al., 1987; Shim et al., 2007). In particular, (Williams et al., 1987; Shim et al., 2007) have observed modes at $251 \mathrm{~cm}^{-1}$ and $280 \mathrm{~cm}^{-1}$ at ambient conditions, which are related to symmetric stretching vibration of the $\mathrm{SiO}_{6}$ octahedral (microstretch behaviour), and octahedral rotation and deformation coupled with displacement of cations in the dodecahedral sites (micropolar behaviour), respectively. Williams et al. (1987) and Shim et al. (2007). At high pressures of $150 \mathrm{GPa}$, modes related to $\mathrm{SiO}_{6}$ octahedral rocking motion (micropolar behaviour) have also been observed at $234 \mathrm{~cm}^{-1}$ (Karki et al., 2000). For our purposes, we thus consider the micro-rotational mode at $234 \mathrm{~cm}^{-1}$, which can be written in terms of frequency

$\omega_{r}[\mathrm{~Hz}]=c_{\text {light }} \omega_{r}\left[\mathrm{~cm}^{-1}\right]=7.015 \times 10^{12} \mathrm{~Hz}$,

where $c_{\text {light }}$ is the speed of light. This value is the cutoff frequency of micro-rotational motions $\omega_{r}$ in bridgmanite (Karki et al., 2000). One can note that the micro-rotational motions reported by Karki et al. (2000) were observed at a higher pressure (150 GPa) compared to 
Table 1

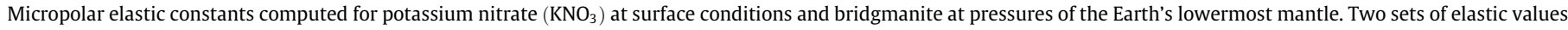
are given for bridgmanite because we have tried two different methods to compute the micro-inertia density $\eta$.

\begin{tabular}{|c|c|c|c|c|c|c|}
\hline Compound & $\mu[\mathrm{GPa}]$ & $\mu_{c}[\mathrm{GPa}]$ & $\mathrm{r}_{\mu_{c}, \mu}$ & $\rho\left[\mathrm{kg} / \mathrm{m}^{3}\right]$ & $\eta[\mathrm{kg} / \mathrm{m}]$ & $L_{c}[\AA]$ \\
\hline $\mathrm{KNO}_{3}$ & 7.175 & 0.025 & 0.00349 & 340.0 & $2.5 \times 10^{-18}$ & 0 \\
\hline bridgmanite (br1) & 310.9 & 1.770 & 0.00569 & 5332 & $1.439 \times 10^{-16}$ & 1.643 \\
\hline bridgmanite (br2) & 310.9 & 0.304 & 0.00098 & 5332 & $2.474 \times 10^{-17}$ & 1.695 \\
\hline
\end{tabular}

Table 2

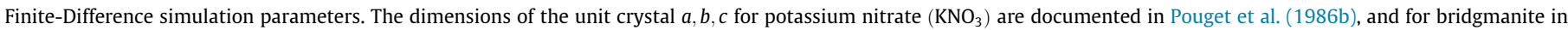

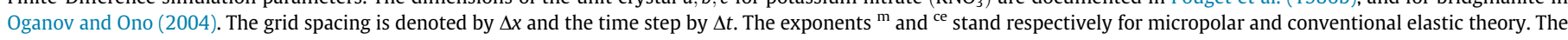

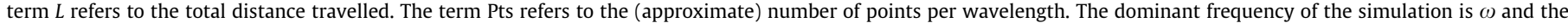
cutoff frequency $\omega_{r}$.

\begin{tabular}{|c|c|c|c|c|c|c|c|c|c|c|}
\hline Compound & $a[\AA]$ & $b[\AA]$ & $c[\AA]$ & $\Delta x[\AA]$ & $(\Delta t)^{m}[s]$ & $(\Delta t)^{c e}[s]$ & $L[\AA]$ & $\omega[\mathrm{Hz}]$ & $\omega_{r}[\mathrm{~Hz}]$ & Pts \\
\hline $\mathrm{KNO}_{3}$ & 7 & 3.75 & 3.24 & $3.75 \times 10^{-4}$ & $8.149 \times 10^{-18}$ & $8.163 \times 10^{-18}$ & 7.5 & $1.23 \times 10^{15}$ & $6.325 \times 10^{12}$ & 100 \\
\hline Bridgmanite & 2.474 & 8.121 & 6.138 & $1.62 \times 10^{-3}$ & $2.121 \times 10^{-17}$ & $2.127 \times 10^{-17}$ & 22.7 & $2.35 \times 10^{15}$ & $2.906 \times 10^{12}$ & 20 \\
\hline
\end{tabular}

a)

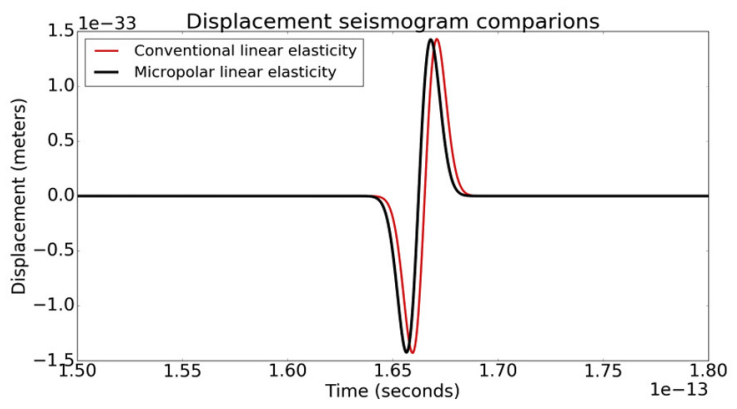

b)

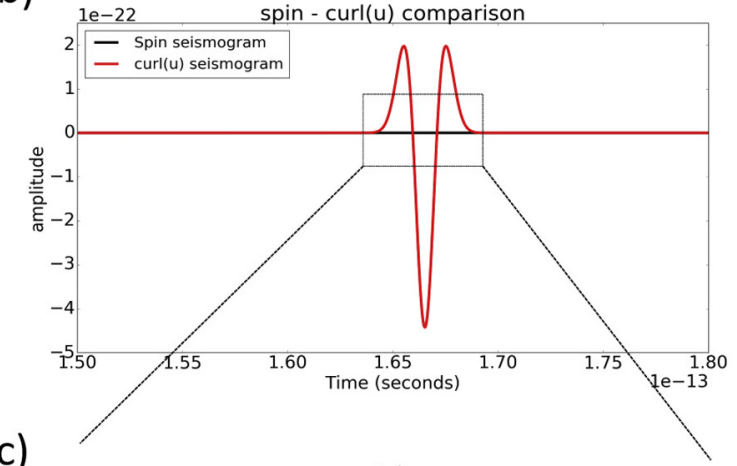

c)

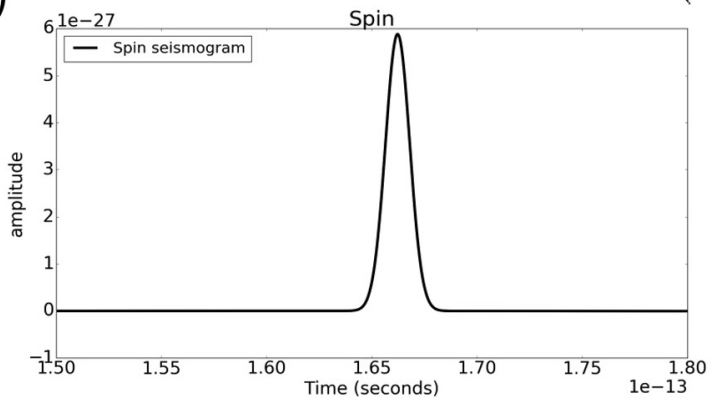

d)

\section{$\mathrm{MgSiO}_{3}$}

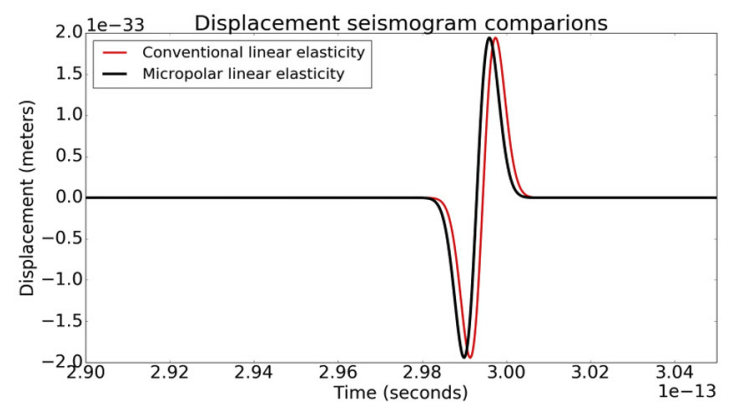

e)

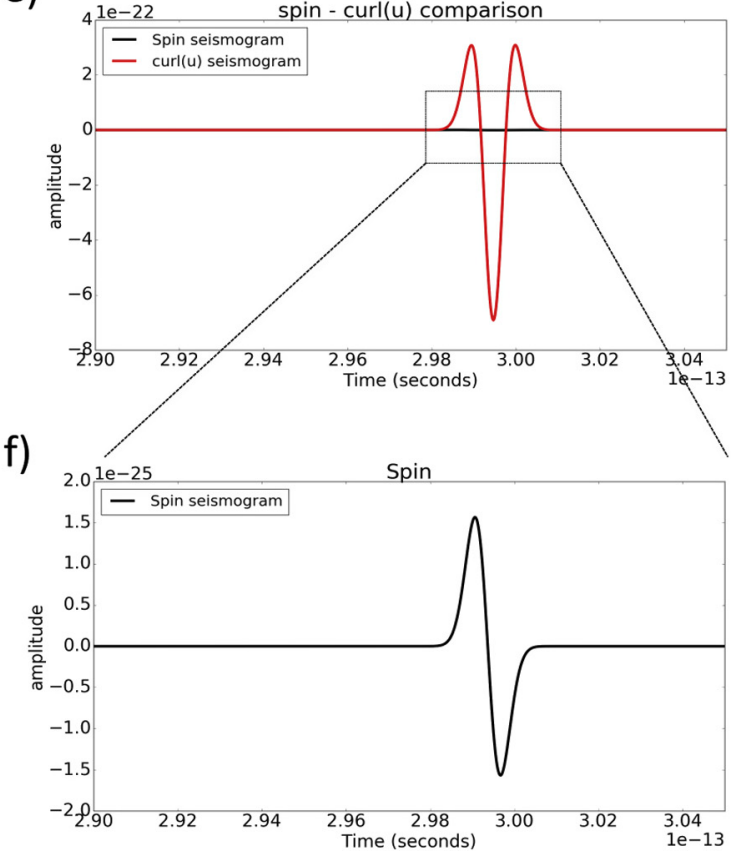

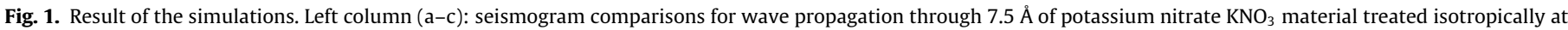

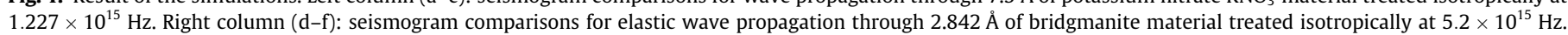

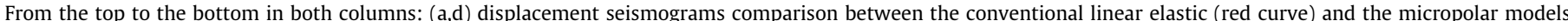

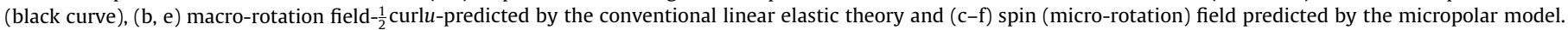
(For interpretation of the references to colour in this figure legend, the reader is referred to the web version of this article.) 


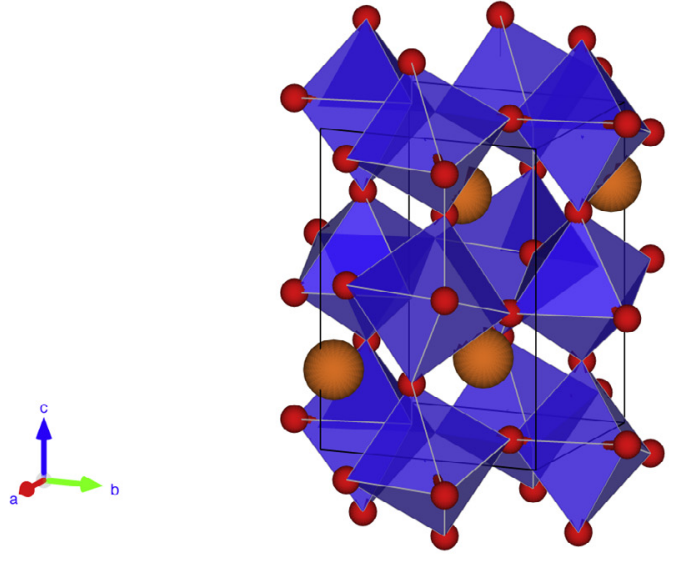

a) Bridgmanite structure

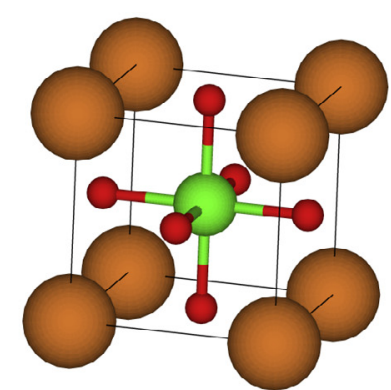

b) Bridgmanite unit cell

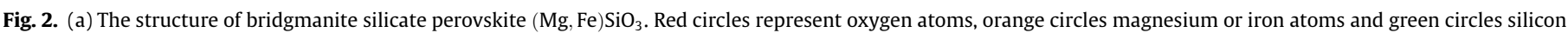

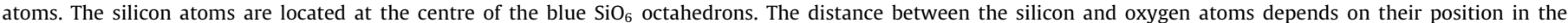

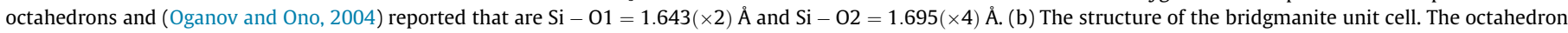

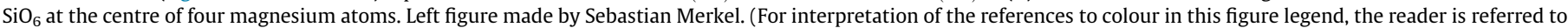
the web version of this article.)

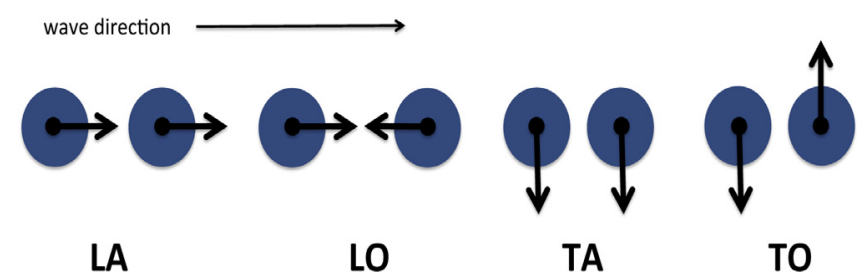

Fig. 3. Typical motions for two atoms in a unit cell, where "L" stands for longitudinal, "T" for transverse, " $\mathrm{A}$ " for acoustic and "O" for optic (after Chen et al., 2004).

the shear modulus $\mu$ found in Oganov and Ono (2004) (120 GPa). However, we show below that the pressure on the Cosserat couple modulus does not significantly affect our results.

In order to compute the Cosserat couple modulus from Eq. (2.2), we still have to estimate the micro-inertia density $\eta$. The determination of the micro-inertia density $\eta$ is a crucial step of the procedure for calculating the Cosserat modulus $\mu_{c}$. A physical definition for $\eta$ is given in Askar (1972), Pouget et al. (1986a), Pouget et al. (1986b), Eringen (1999), Nowacki (1986), as follows

$\eta=\frac{j}{V}$

where $j$ is the conventional micro-inertia (moment of inertia) and $V$ is the considered volume.

Here we are interested in the micro-motions of the unit $\mathrm{MgSiO}_{3}$ cell. In order to compute the moment of micro-inertia $j$ related to the rotation of the molecular group $\mathrm{SiO}_{6}$, we assume that the rotational motion occurs with respect to the silicon and two oxygen atoms. The moment of micro-inertia $j$ can then be found as follows

$j=\frac{4 \mathrm{M}_{\mathrm{O}}}{\mathrm{N}_{\mathrm{A}}} \cdot(\mathrm{Si}-\mathrm{O} 2)^{2}=3.051 \times 10^{-45} \mathrm{~kg} \mathrm{~m}^{2}$,

where $\mathrm{N}_{\mathrm{A}}$ is the Avogadro's constant, $\mathrm{Si}-\mathrm{O} 2$ the distance of the oxygen atoms to the silicon atom and $\mathrm{M}_{\mathrm{O}}$ the molecular weight of the oxygen, $15.99 \mathrm{~g} \mathrm{~mol}^{-1}$. The micro-inertia density $\eta$ is then found by

$\eta=\frac{j}{V}=2.474 \times 10^{-17} \mathrm{~kg} \mathrm{~m}^{-1}$,

with $\mathrm{V}=a \times b \times c=1.23310^{-28} \mathrm{~m}^{3}$,

the volume of the unit cell.
These calculations finally yield a Cosserat couple modulus of $0.304 \mathrm{GPa}$ that we will use in our simulations. One should note that we could also have used Eq. (2.4) to compute the micro-inertia density $\eta$. This would imply to assume some characteristic length $L_{c}$. A reasonable characteristic length could be the distance between the silicon and oxygen atoms $\mathrm{Si}-\mathrm{O} 1=1.643 \AA$ (Oganov and Ono, 2004), which gives $\eta=1.439 \times 10^{-16} \mathrm{~kg} \mathrm{~m}^{-1}$ and a Cosserat couple modulus of $1.77 \mathrm{GPa}$. The results for both calculations are summarized in Table 1.

Table 2 gives the simulation parameters. We again consider a Ricker source time function located in the displacement field only (see Appendix B). Fig. 1(d-f) shows the obtained results. We observe that there exists a visible time lag (less than $1 \mathrm{~s}$ ) between both time series obtained with the linear micropolar and conventional linear elastic theories (Fig. 1-d). Moreover, as in the previous simulation, the spin field $\vartheta$ shows smaller amplitude and different waveform compared to the rotational field predicted by the conventional linear elastic theory (Fig. 1-e-f). We find from our numerical experiments that the conventional linear elastic and micropolar models no longer agree in their predictions of linear elastic wave propagation.

\section{Dispersion analysis}

In order to perform a dispersion analysis in the case of micropolar theory, we assume plane wave solutions for the equations of motion of the form

$(u(x, t), \vartheta(x, t))=\left(\gamma^{1}, \gamma^{2}\right) e^{\mathbf{i}(k x-\omega t)}$,

where $\bar{\gamma}=\left(\gamma^{1}, \gamma^{2}\right)$ are the unknown amplitudes of the considered waves. Substituting Eq. (4.9) in the micropolar equations of motion Eq. (2.2), we obtain the following expression

$$
\mathscr{A} \cdot \bar{\gamma}=0 \quad \text { with } \quad \mathscr{A}=\left[\begin{array}{cc}
\rho \omega^{2}-\left(\mu+\mu_{c}\right) k^{2}- & \mathbf{i} k 2 \mu_{c} \\
\mathbf{i} k 2 \mu_{c} & \eta \omega^{2}-\mu L_{c}^{2} k^{2}-4 \mu_{c}
\end{array}\right] .
$$

In order to obtain non-trivial solutions $(\bar{\gamma} \neq 0)$ of the algebraic system in Eq. (4.10), one must impose that

$\operatorname{det} \mathscr{A}=0$. 
Eq. (4.11) is called the dispersion relation $\omega(k)$ for a micropolar model. From Eq. (4.11) we obtain explicit expressions for the frequency $\omega$ and phase velocity $c=\frac{\omega}{k}$ given by the following expressions

$\omega= \pm \sqrt{\frac{\left[\left(\frac{\mu L_{c}^{2}}{\eta}+\frac{\mu+\mu_{c}}{\rho}\right) k^{2}+\omega_{r}^{2}\right] \pm \sqrt{\left[\left(\frac{\mu L_{c}^{2}}{\eta}+\frac{\mu+\mu_{c}}{\rho}\right) k^{2}+\omega_{r}^{2}\right]^{2}-4 k^{2}\left[\left(\frac{\mu+\mu_{c}}{\rho}\right)\left(\frac{\mu \mu_{c}^{2}}{\eta}\right) k^{2}+\omega_{r}^{2} \frac{\mu}{\rho}\right]}}{2}}$,

$\begin{aligned} c & =\frac{\omega}{k} \\ & = \pm \sqrt{\frac{\left[\frac{\mu_{c}}{\rho}+\frac{\mu L_{c}^{2}}{\eta}+\left(1-\frac{\omega_{r}^{2}}{\omega^{2}}\right) \frac{\mu}{\rho}\right] \pm \sqrt{\left[\frac{\mu_{c}}{\rho}+\frac{\mu L_{c}^{2}}{\eta}+\left(1-\frac{\omega_{c}^{2}}{\omega^{2}}\right) \frac{\mu}{\rho}\right]^{2}-4\left(1-\frac{\omega_{c}^{2}}{\omega^{2}}\right)\left(\frac{\mu+\mu_{c}}{\rho}\right)\left(\frac{\mu L_{c}^{2}}{\eta}\right)}}{2\left(1-\frac{\omega_{t}^{2}}{\omega^{2}}\right)}} .\end{aligned}$

We find that there are four possible phase velocities $\left(c_{1},-c_{1}, c_{2},-c_{2}\right)$ which correspond to four wavenumbers $\left(k_{1},-k_{1}, k_{2},-k_{2}\right)$, which means that there are four waves that are solution of the micropolar wave equation, two in each directions. Therefore, the solutions in displacement and spin $(u(x, t), \vartheta(x, t))$ are of the form

$$
\begin{aligned}
u(x, t) & =A_{-} e^{\mathbf{i}\left(\omega t-k_{1} x\right)}+A_{+} e^{\mathbf{i}\left(\omega t+k_{1} x\right)}+B_{-} e^{\mathbf{i}\left(\omega t-k_{2} x\right)}+B_{+} e^{\mathbf{i}\left(\omega t+k_{2} x\right)}, \\
& =A_{-} e^{\mathbf{i} k_{1}\left(c_{1} t-x\right)}+A_{+} e^{\mathbf{i} k_{1}\left(c_{1} t+x\right)}+B_{-} e^{\mathbf{i} k_{2}\left(c_{2} t-x\right)}+B_{+} e^{\mathbf{i} k_{2}\left(c_{2} t+x\right)} \\
\vartheta(x, t) & =C_{-} e^{\mathbf{i}\left(\omega t-k_{1} x\right)}+C_{+} e^{\mathbf{i}\left(\omega t+k_{1} x\right)}+D_{-} e^{\mathbf{i}\left(\omega t-k_{2} x\right)}+D_{+} e^{\mathbf{i}\left(\omega t+k_{2} x\right)}, \\
& =C_{-} e^{\mathbf{i} k_{1}\left(c_{1} t-x\right)}+C_{+} e^{\mathbf{i} k_{1}\left(c_{1} t+x\right)}+D_{-} e^{\mathbf{i} k_{2}\left(c_{2} t-x\right)}+D_{+} e^{\mathbf{i} k_{2}\left(c_{2} t+x\right)}
\end{aligned}
$$

where $A_{+}, A_{-}, B_{+}, B_{-}, C_{+}, C_{-}, D_{+}, D_{-}$are the waveform amplitudes that need to be determined from the boundary conditions of the problem. The phase velocities $c_{1}, c_{2}$ are frequency dependent, unlike in the linear elastic theory. It is interesting to consider the case $L_{c}=0$, called reduced Cosserat model (Kulesh et al., 2009; Grekova et al., 2009; Grekova, 2012a,b, 2016), which gives from (4.13)

$$
c^{2}=c_{T}^{2} \frac{1-\omega^{2} / \omega_{1}^{2}}{1-\omega^{2} / \omega_{r}^{2}} \quad \text { with } \quad c_{T}=\sqrt{\frac{\mu}{\rho}}, \quad \omega_{1}=\frac{\omega_{r}}{\sqrt{1+\frac{\mu_{c}}{\mu}}},
$$

This equation tells us that the phase velocity is zero for $\omega=\omega_{1}$, meaning there is no wave propagation, and that the phase velocity goes to infinity for $\omega=\omega_{r}$. These two frequencies actually define a frequency gap between $\omega_{1}$ and $\omega_{r}$ where there is no wave propagation. Moreover, it is noteworthy that for $L_{c}=0$ and for $\omega \gg \omega_{r}$, as used in our simulations in potassium nitrate, we find only two phase velocities

$c=\frac{\omega}{k}= \pm \sqrt{\frac{\mu+\mu_{c}}{\rho}}$

This phase velocity tells us that, for this particular case, the difference between the linear micropolar and conventional linear elastic model is given by the micropolar couple modulus $\mu_{c}$.

In Fig. 4 we display the obtained micropolar phase velocity as a function of the frequency $\omega$ for potassium nitrate (panel a) and bridgmanite (panel b). As a matter of simplicity we only represent the positive phase velocities (those referred as $c_{1}$ and $c_{2}$ ). Note that in the case of potassium nitrate the characteristic length is assumed to be zero, which gives a completely different behaviour compared to when $L_{c} \neq 0$. We can observe on panel (a) the frequency band gap predicted for $L_{c}=0$ which does not appear for bridgmanite. We observe, as expected from Eq. (4.13), that there exists an indetermination at $\omega=\omega_{r}$, meaning that there is no micropolar wave propagation at $\omega=\omega_{r}$. The acoustic mode shows only real phase velocity values for the entire frequency range. Its behaviour is almost linear with frequency unlike the optic mode $\vartheta$ which varies significantly. This means that the acoustic mode is almost frequency independent and the optic mode shows only real velocity values for $\omega>\omega_{r}$. Imaginary values of the phase velocities are not related to dissipation effects but to localization phenomena $^{1}$ (Grekova et al. (2009)). Also note that even if one of the phase speeds $\left(c_{1}, c_{2}\right)$ is zero, it does not imply that there is no wave propagation since both speeds are coupled (see Eq. 4.14). We now look at the same equations but plotting the frequency $\omega$ as a function of wavenumber $k$, as presented in Fig. 5. It illustrates micropolar and conventional dispersion curves obtained for potassium nitrate and bridgmanite crystals. We observe that the acoustic modes computed with the conventional and micropolar models are the same. However, as observed in laboratory experiments, the micro-rotational mode (optic mode) occurs at much higher frequencies, evidencing the presence of a frequency band gap between the acoustic $u$ and optic $\vartheta$ modes in the case of potassium nitrate (Fig. 4 panel a) and which becomes only a frequency singularity in the general case, as for bridgmanite (Fig. 4 panel b).

We thus show that unlike the conventional linear elastic wave propagation, in the case of the shear micropolar wave propagation (Eq. (2.2)) dispersion effects, frequency band gaps (Abreu et al., 2016) and cutoff frequencies can be predicted. Both phenomena have been observed in Raman spectroscopy experiments conducted on $\mathrm{MgSiO}_{3}$ silicate perovskite (Durben and Wolf, 1992; Hirai et al., 2011; Ghose et al., 1994). However, because the optic modes are at much higher frequencies compared to the acoustic modes, their existence has been often considered as a secondgradient elastic effect. We show in this study that it is possible to account for these effects by applying the micropolar theory, and the measurement of the observed cutoff frequency $\omega_{r}$ can provide constraints on the Cosserat couple modulus and yield more insights into the micropolar elastic properties in real materials.

We thus now propose to use the measured cut-off frequencies for bridgmanite at various pressures and temperatures in order to derive the Cosserat couple modulus for the same range of temperatures and pressures which will enable us to apply micropolar theory to deep Earth-like material. To do so, we use the critical frequency $\omega_{r}$ values found in Bukowinski et al. (1996) and Parlinski and Kawazoe (2000) to determine the behaviour of the Cosserat couple modulus $\mu_{c}$ of bridgmanite as a function of temperature and pressure. For the calculations of the Cosserat couple modulus $\mu_{c}$, we assume constant density of $\rho=5331.98 \mathrm{~kg} / \mathrm{m}^{3}$ and a characteristic length of $L_{c}=1.643 \AA$. The Cosserat modulus is computed applying Eq. (2.3) and the results are displayed in Fig. 6. It appears that for a fixed temperature, the Cosserat couple modulus $\mu_{c}$ increases with pressure (Fig. 6 left), and that for a fixed pressure, the Cosserat couple modulus $\mu_{c}$ decreases with temperature (Fig. 6 right). We actually expect this pressure-temperature behaviour since at higher temperatures and lower pressures the molecules vibrate more energetically, making the material easier to deform and so leading to lower Cosserat couple moduli. We can also observe that the difference in the values of the Cosserat couple modulus for pressures between $120 \mathrm{GPa}$ and $150 \mathrm{GPa}$ is small. This justifies the fact that in our simulations we keep the values of Cosserat couple modulus at $150 \mathrm{GPa}$ compared to the value of the shear modulus. We finally provide a catalogue in Table 3 of the values of the Cosserat couple modulus $\mu_{c}$ shown in Fig. 6.

\footnotetext{
1 Localization phenomena or more generally strain localization refers to a narrow zone of intense shear strain developed during the deformation process
} 


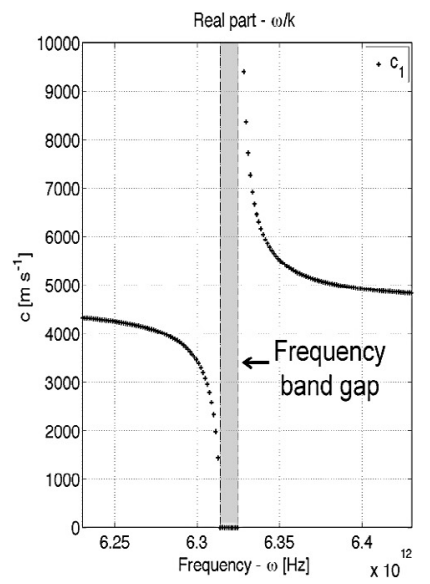

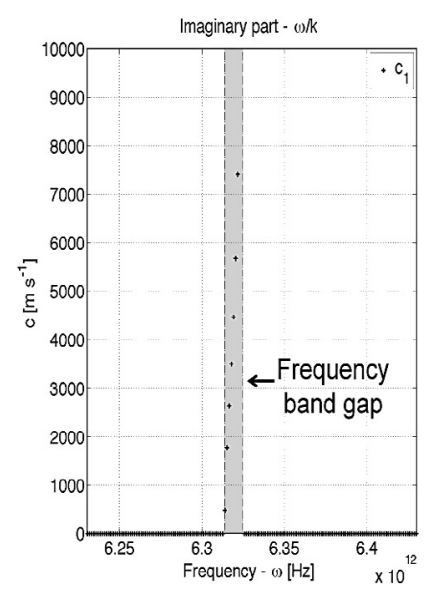

a) Potassium nitrate
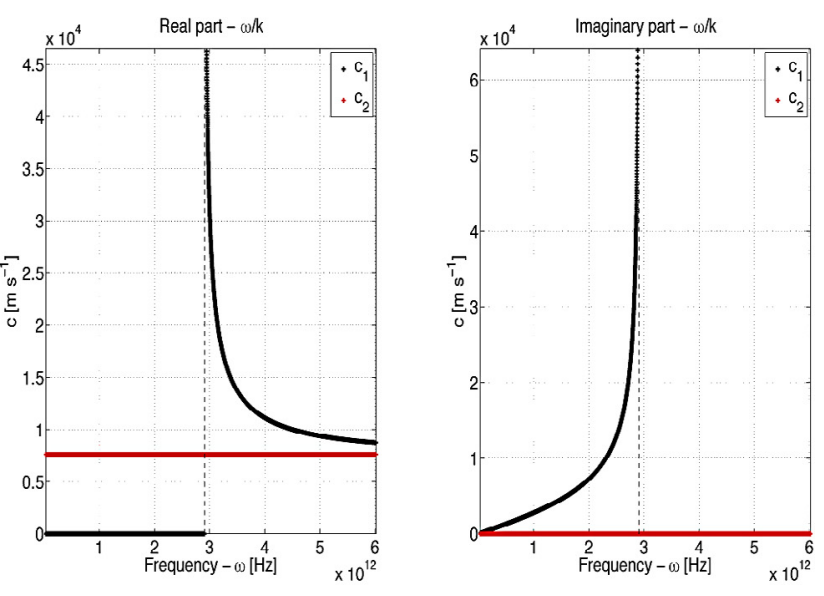

\section{b) Bridgmanite}

Fig. 4. Real and imaginary parts of the two phase velocities $c_{1}, c_{2}$ in (a) potassium nitrate and (b) bridgmanite.

a)

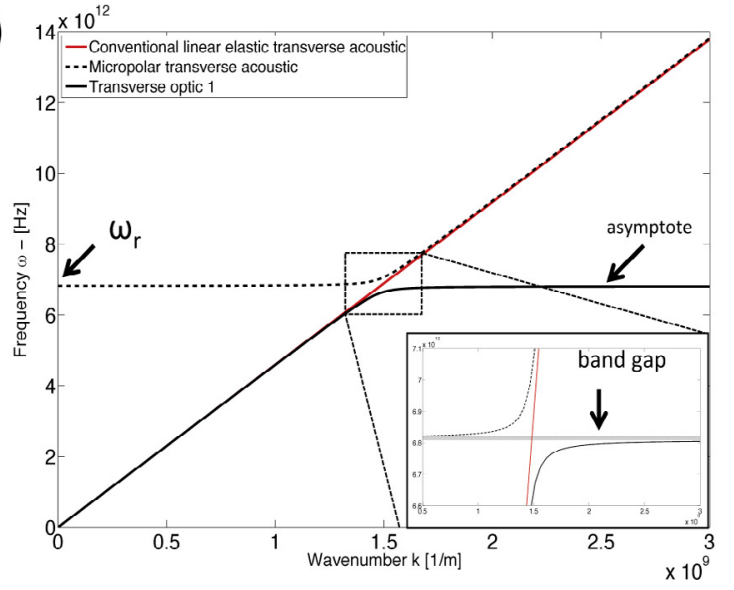

b)

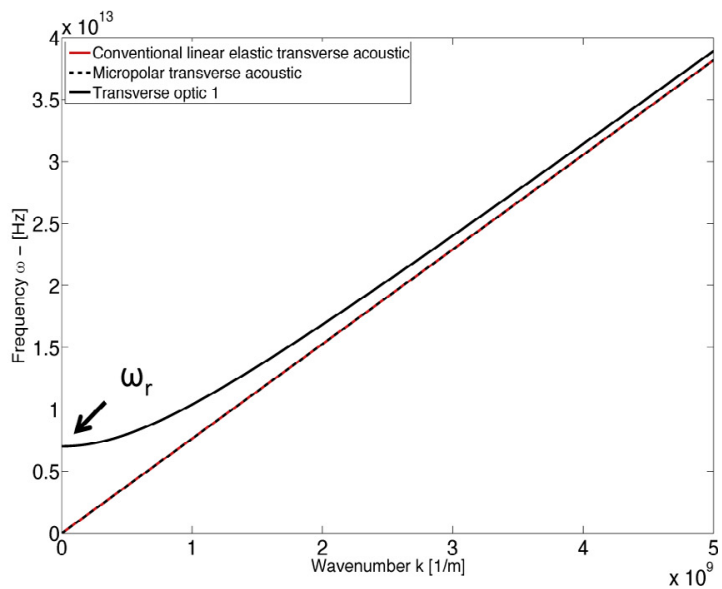

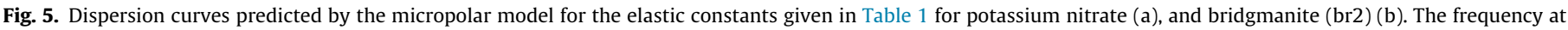
which the transverse optic modes occur is $\omega_{r}$, different in both cases and given in Table 1.
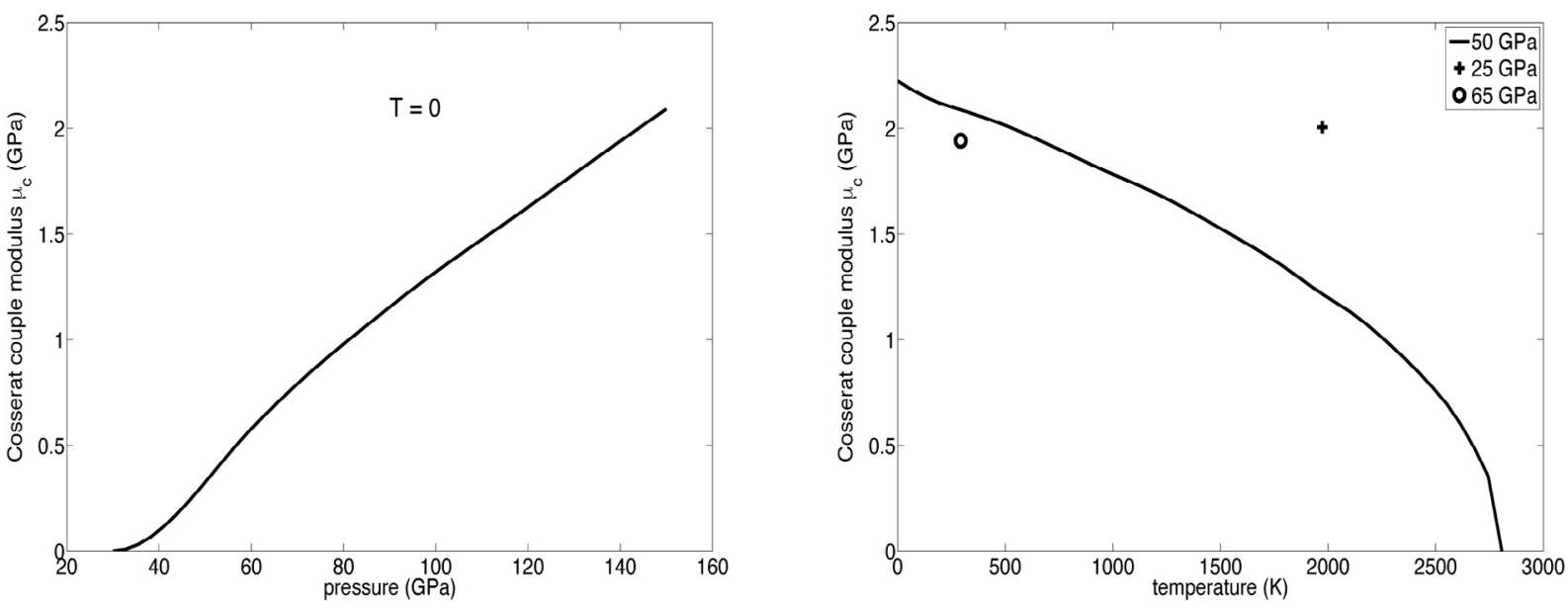

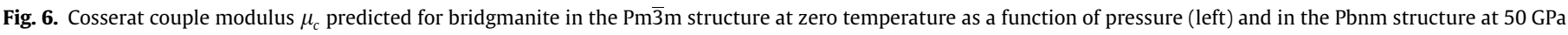

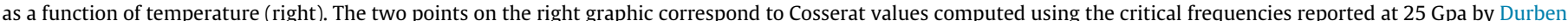

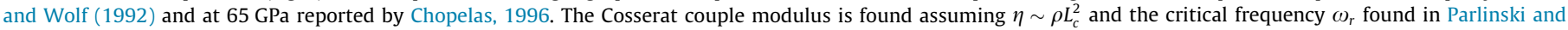
Kawazoe (2000) (left) and Bukowinski et al. (1996) (right). 
Table 3

Values of the Cosserat couple modulus $\mu_{c}$ as a function of pressure at ambient conditions $(\mathrm{T}=0)$ and as a function of temperature at $50 \mathrm{GPa}$.

\begin{tabular}{|c|c|c|c|}
\hline Pressure $[\mathrm{GPa}]$ at $\mathrm{T}=0$ & $\mu_{c}[\mathrm{GPa}]$ & Temperature $[\mathrm{K}]$ at $50 \mathrm{GPa}$ & $\mu_{c}[\mathrm{GPa}]$ \\
\hline 30.006 & 0.000 & 0.000 & 2.224 \\
\hline 34.145 & 0.019 & 96.812 & 2.165 \\
\hline 38.283 & 0.069 & 193.624 & 2.120 \\
\hline 42.422 & 0.144 & 290.436 & 2.088 \\
\hline 46.561 & 0.238 & 387.247 & 2.056 \\
\hline 50.699 & 0.344 & 484.059 & 2.021 \\
\hline 54.838 & 0.452 & 580.871 & 1.980 \\
\hline 58.977 & 0.555 & 677.683 & 1.934 \\
\hline 63.116 & 0.648 & 774.495 & 1.889 \\
\hline 67.254 & 0.736 & 871.307 & 1.842 \\
\hline 71.393 & 0.819 & 968.119 & 1.797 \\
\hline 75.532 & 0.898 & 1064.931 & 1.754 \\
\hline 79.670 & 0.973 & 1161.742 & 1.711 \\
\hline 83.809 & 1.046 & 1258.554 & 1.663 \\
\hline 87.948 & 1.118 & 1355.366 & 1.610 \\
\hline 92.086 & 1.190 & 1452.178 & 1.554 \\
\hline 96.225 & 1.260 & 1548.990 & 1.497 \\
\hline 100.364 & 1.326 & 1645.802 & 1.440 \\
\hline 104.503 & 1.391 & 1742.614 & 1.380 \\
\hline 108.641 & 1.454 & 1839.426 & 1.313 \\
\hline 112.780 & 1.516 & 1936.237 & 1.242 \\
\hline 116.919 & 1.579 & 2033.049 & 1.177 \\
\hline 121.057 & 1.644 & 2129.861 & 1.112 \\
\hline 125.196 & 1.708 & 2226.673 & 1.032 \\
\hline 129.335 & 1.773 & 2323.485 & 0.943 \\
\hline 133.474 & 1.838 & 2420.297 & 0.846 \\
\hline 137.612 & 1.902 & 2517.109 & 0.738 \\
\hline 141.751 & 1.966 & 2613.920 & 0.604 \\
\hline 145.890 & 2.029 & 2710.732 & 0.423 \\
\hline 150.028 & 2.092 & 2807.544 & 0.000 \\
\hline
\end{tabular}

\section{Discussions and concluding remarks}

Until today the main applications of micropolar theory in seismic studies have been related to the modelling the elastic response of granular medium (Twiss et al., 1993; Schwartz and Johnson, 1984; Mouraille et al., 2006; Hiraiwa et al., 2016; Merkel and Luding, 2017; Merkel et al., 2011) or to better describe brittle deformation at crustal scale (Kissel and Laj, 2012; Luyendyk et al., 1980; Wells and Heller, 1988). In particular, it has been shown that it plays a key role in rupture processes at plate tectonics scale (Twiss, 2009; Twiss et al., 1993; Gade and Raghukanth, 2016; Teisseyre et al., 2006). This comes from the incapability of the linear elastic theory to provide a complete continuum description of distributed brittle deformation (Twiss et al., 1993). It is thus believed that the correct finding and physical interpretation of elastic constants in micro-continuum theories can help to better understand plate tectonic processes, and should for instance be taken into account for studying the initiation of plate tectonics (Bercovici and Ricard, 2014; Bercovici and Ricard, 2012). However, the detailed effects of micropolar media on seismic wave propagation at seismic periods, $\sim 1 \mathrm{~s}$, and large scales, greater than $100 \mathrm{~km}$, have not yet been reported; this is mainly due to the lack of micropolar elastic constants of deep mantle materials necessary to implement the micropolar theory.

We have focused on the description of the wave propagation including micro rotational effects for the potassium nitrate $\mathrm{KNO}_{3}$ and perovskite crystal structures at ultrasonic frequencies. Using micropolar elastic constants found in the literature for potassium nitrate (Eringen, 1999) and computed for bridgmanite using measurements of laboratory observations from Hemley et al. (1987), Karki et al. (2000), Durben and Wolf (1992), Chopelas (1996), Williams et al. (1987) and Shim et al. (2007), we have performed numerical simulations using the Finite-Difference method and compared the micropolar behaviour with respect to the conventional linear elastic behaviour. For the high frequencies used here, micropolar wave propagation predicts faster displacement waves with respect to the linear elastic model and a reduction in amplitude, similar to attenuation effects observed in the Earth (e.g. Romanowicz and Mitchell, 2007). These marked differences between linear elastic and micropolar theories should now be carefully explored when considering a larger body such as the Earth.

We show that micropolar theory offers the opportunity to relate acoustic and optic modes with the cutoff frequency observed in Raman spectroscopy experiments (Eringen, 1999). Utilizing data obtained from various experiments that have observed the optic modes in bridgmanite (Hemley et al., 1987; Karki et al., 2000; Durben and Wolf, 1992; Chopelas, 1996; Williams et al., 1987; Shim et al., 2007), we were able to compute Cosserat couple modulus $\mu_{c}$ for bridgmanite at various pressures and temperatures, providing thus a catalogue of possible values that can be used in future work to simulate micropolar motions in deep Earth's mantle-like materials and to test the influence of microrotational motions on the seismic wavefield.

As shown here, the micropolar theory offers the opportunity of describing the elastic dynamics of atoms in a crystal. The dynamic characteristic of a crystal depends on the crystal's structure and the binding between the atoms. It is the atom's dynamics and arrangement that controls macroscopic effect on seismic wave propagation. For instance, as stated before, the dynamics of the $\mathrm{SiO}_{6}$ octahedral at very high frequencies can change the crystal's structure. This lattice dynamics may induce phase transitions (Cowley, 1964). Further 3D numerical experiments are needed to study whether the observed micropolar motions lead to permanent changes in the crystal structure of bridgmanite that will be related to changes in seismic wave speeds and attenuation effects and can be observed and analysed.

Not only attenuation effects may be due to micropolar motions (Romeo, 2015): it has be shown by Cordier et al. (2014) that using a general description of deformation processes including rotational defects can help to predict slip systems in olivine. Hence, the micropolar theory in combination with calibrated laboratory studies, can thus help to better constrain the lattice dynamics and deformation styles in the lower mantle.

It is possible to change scales from micro to macro-scale effects using micropolar media through the characteristic length $L_{c}$ parameter. For our purposes of wave propagation at the crystal scale, we assumed a very small characteristic length scale, of the order of atomic distances. However, if investigating the effect of micropolar motions at meso- to macro-scale, one has to re-consider this characteristic scale-length. For this one has to compute, using Eq. (2.3) and Eq. (2.4), the obtained $\omega_{r}$ range for a range of characteristic length $L_{c}$. Taking the values of Cosserat couple modulus and shear modulus given Table 1, last row (br2), we obtain Fig. 7. Since linear elasticity theory provides reasonable results for simulating wave propagation and matching waveforms, travel times and amplitudes of seismic waves for teleseismic studies, we do not expect $\omega_{r}$ to be in the seismic period band for teleseismic waves of 1-100 s. Excluding these values yields an upper bound for the characteristic length that should be smaller than $200 \mathrm{~km}$ for the lower mantle. Several structures in the lower mantle are of the order of $200 \mathrm{~km}$ length, however, the most likely effect would be at the grain size level. Recently (Solomatov et al., 2008) propose that grain sizes in the lower mantle are of the order of $1 \mathrm{~mm}$ to $1 \mathrm{~m}$, which is well below our upper bound. Heterogeneity, such as ultra-low velocity zones (Thorne and Garnero, 2004), which could be due to a certain degree of melt (Hier-Majumder, 2014; Liu et al., 2016), is unlikely to be affected by micropolar motions, as they would not rotate independently of the surrounding mantle. 


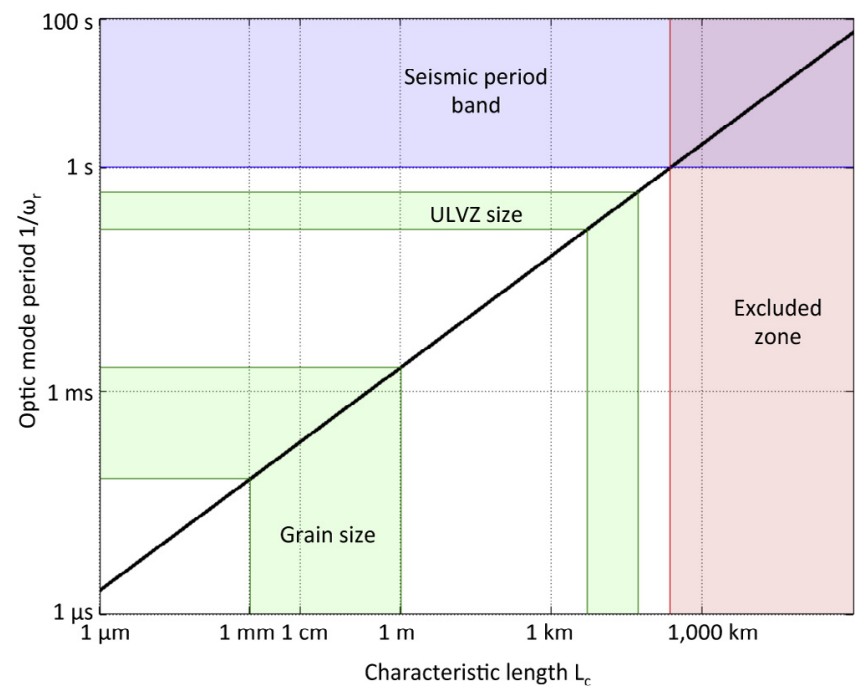

Fig. 7. Obtained optic mode periods $\left(1 / \omega_{r}\right)$ in bridgmanite in lower mantle conditions as a function of characteristic length $L_{c}$. Excluding periods in the seismic frequency band yields a constraint on $L_{c}$ that must be smaller than $\sim 200 \mathrm{~km}$.

There is hope in the future to compare simulations of wave propagation in micropolar media with seismological data such as rotational records provided by the emerging technology of rotational sensors (Yin et al., 2016; Gade and Raghukanth, 2016; Suryanto et al., 2006; Schreiber et al., 2006; Bernauer et al., 2009; Ferreira and Igel, 2009; Wassermann et al., 2009; Igel et al., 2007, 2005). It has already been shown that in the nearfield, rotational ground motions are much larger compared to what is expected from the classical linear elasticity (Lee et al., 2009). Only the combination of seismological data with numerical simulations will help us to answer the question whether micropolar theory can better explain travel times (e.g. Thorne et al., 2013; Garnero and Helmberger, 1993; Zaroli et al., 2010) and/or attenuation anomalies observed by seismologists in the Earth (Durek and Ekström, 1996; Widmer et al., 1991; Lawrence and Wysession, 2006; Hwang and Ritsema, 2011; Durand et al., 2013).

Finally, this study arrives to the question of how extrapolating the measurements of elastic parameters made in laboratory experiments at crystal scale (micro) to our length scales in seismology (macro). As mentioned above, linear elastic theory does describe the wave propagation on a global scale well but amplitude and travel time effects are observed in our small-scale modelling of micropolar media. Those effects are indeed present in minerals (Takei et al., 2011) and the measurements of wave propagation on those small-scale experiments have not been easily be related to the Earth, as a simple scaling from the microscopic scale (mineral) to the macroscopic scale (Earth). Further, detailed numerical experiments are needed and have to be compared with laboratory experiments to understand scaling between those effects.

\section{Acknowledgements}

We would like to thank the Editor Michael Le Bars and one anonymous reviewer for helpful comments that improved the manuscript. R.A. was partly supported by the Spanish Ministry of
Economy and Competitiveness (project CGL2015-67130-C2-2-R). R.A. acknowledges initial comments of Jeroen Tromp on micropolar media, Patrizio Neff on micro-continuum field theories and Michael Slawinski for useful comments on the isotropic approximation of general anisotropic elasticity tensors. Fig. 2 was provided by Sebastian Merkel. S.D. is supported by DFG grant HAADES DU1634/1-1.

\section{Appendix A. Closest isotropic micropolar tensor}

The elastic constants for potassium nitrate $\mathrm{KNO}_{3}$ given in Eringen (1999) are recalled in Table 4 (see Eringen, 1999 page $160)$. In order to make comparison with the conventional linear elastic model, we compute the isotropic approximation to the orthorhombic crystal structure given in Eringen (1999) (and recalled in Table 4). Following the definition of the nonsymmetric micropolar force stress tensor $\sigma(2.1)$ in general anisotropic media, we can write

$\sigma_{i j}=\sum_{k=1}^{3} \sum_{l=1}^{3} \mathbb{C}_{i j k l} e_{k l} \quad$ with $\quad i, j \in\{1,2,3\}$,

were $\mathbb{C}$ is the fourth order tensor of elastic constants and $e$ the nonsymmetric second order micropolar strain tensor (2.1). We can express (A.17) in a matrix form as follows

$\left(\begin{array}{l}\sigma_{11} \\ \sigma_{22} \\ \sigma_{33} \\ \sigma_{23} \\ \sigma_{13} \\ \sigma_{12} \\ \sigma_{32} \\ \sigma_{31} \\ \sigma_{21}\end{array}\right)=\left(\begin{array}{lllllllll}\mathbb{C}_{1111} & \mathbb{C}_{1122} & \mathbb{C}_{1133} & \mathbb{C}_{1123} & \mathbb{C}_{1113} & \mathbb{C}_{1112} & \mathbb{C}_{1132} & \mathbb{C}_{1131} & \mathbb{C}_{1121} \\ \mathbb{C}_{2211} & \mathbb{C}_{2222} & \mathbb{C}_{2233} & \mathbb{C}_{2223} & \mathbb{C}_{2213} & \mathbb{C}_{2212} & \mathbb{C}_{2232} & \mathbb{C}_{2231} & \mathbb{C}_{2221} \\ \mathbb{C}_{3311} & \mathbb{C}_{3322} & \mathbb{C}_{3333} & \mathbb{C}_{3323} & \mathbb{C}_{3313} & \mathbb{C}_{3312} & \mathbb{C}_{3332} & \mathbb{C}_{3331} & \mathbb{C}_{3321} \\ \mathbb{C}_{2311} & \mathbb{C}_{2322} & \mathbb{C}_{2333} & \mathbb{C}_{2323} & \mathbb{C}_{2313} & \mathbb{C}_{2312} & \mathbb{C}_{2332} & \mathbb{C}_{2331} & \mathbb{C}_{2321} \\ \mathbb{C}_{1311} & \mathbb{C}_{1322} & \mathbb{C}_{1333} & \mathbb{C}_{1323} & \mathbb{C}_{1313} & \mathbb{C}_{1312} & \mathbb{C}_{1332} & \mathbb{C}_{1331} & \mathbb{C}_{1321} \\ \mathbb{C}_{1211} & \mathbb{C}_{1222} & \mathbb{C}_{1233} & \mathbb{C}_{1223} & \mathbb{C}_{1213} & \mathbb{C}_{1212} & \mathbb{C}_{1232} & \mathbb{C}_{1231} & \mathbb{C}_{1221} \\ \mathbb{C}_{3211} & \mathbb{C}_{3222} & \mathbb{C}_{3233} & \mathbb{C}_{3223} & \mathbb{C}_{3213} & \mathbb{C}_{3212} & \mathbb{C}_{3232} & \mathbb{C}_{3231} & \mathbb{C}_{3221} \\ \mathbb{C}_{3111} & \mathbb{C}_{3122} & \mathbb{C}_{3133} & \mathbb{C}_{3123} & \mathbb{C}_{3113} & \mathbb{C}_{3112} & \mathbb{C}_{3132} & \mathbb{C}_{3131} & \mathbb{C}_{3121} \\ \mathbb{C}_{2111} & \mathbb{C}_{2122} & \mathbb{C}_{2133} & \mathbb{C}_{2123} & \mathbb{C}_{2113} & \mathbb{C}_{2112} & \mathbb{C}_{2132} & \mathbb{C}_{2131} & \mathbb{C}_{2121}\end{array}\right)\left(\begin{array}{l}e_{11} \\ e_{22} \\ e_{33} \\ e_{23} \\ e_{13} \\ e_{12} \\ e_{32} \\ e_{31} \\ e_{21}\end{array}\right)$

In isotropic media we can write the non-symmetric micropolar force stress tensor as follows

$\sigma_{i j}=\lambda \sum_{k=1}^{3} \delta_{i j} e_{k k}+\left(\mu+\mu_{c}\right) e_{i j}+\left(\mu-\mu_{c}\right) e_{j i}$,

where $\lambda, \mu$ and $\mu_{c}$ are the conventional Lamé parameters and the Cosserat couple modulus, respectively. We can write in matrix notation the following (see Zheng and Spencer, 1993)

$\left(\begin{array}{c}\sigma_{11} \\ \sigma_{22} \\ \sigma_{33} \\ \sigma_{23} \\ \sigma_{13} \\ \sigma_{12} \\ \sigma_{32} \\ \sigma_{31} \\ \sigma_{21}\end{array}\right)=\left(\begin{array}{ccccccccc}\lambda+2 \mu & \lambda & \lambda & 0 & 0 & 0 & 0 & 0 & 0 \\ \lambda & \lambda+2 \mu & \lambda & 0 & 0 & 0 & 0 & 0 & 0 \\ \lambda & \lambda & \lambda+2 \mu & 0 & 0 & 0 & 0 & 0 & 0 \\ 0 & 0 & 0 & \mu+\mu_{c} & 0 & 0 & \mu-\mu_{c} & 0 & 0 \\ 0 & 0 & 0 & 0 & \mu+\mu_{c} & 0 & 0 & \mu-\mu_{c} & 0 \\ 0 & 0 & 0 & 0 & 0 & \mu+\mu_{c} & 0 & 0 & \mu-\mu_{c} \\ 0 & 0 & 0 & \mu-\mu_{c} & 0 & 0 & \mu+\mu_{c} & 0 & 0 \\ 0 & 0 & 0 & 0 & \mu-\mu_{c} & 0 & 0 & \mu+\mu_{c} & 0 \\ 0 & 0 & 0 & 0 & 0 & \mu-\mu_{c} & 0 & 0 & \mu+\mu_{c}\end{array}\right)\left(\begin{array}{l}e_{11} \\ e_{22} \\ e_{33} \\ e_{23} \\ e_{13} \\ e_{12} \\ e_{32} \\ e_{31} \\ e_{21}\end{array}\right)$.

In order to find the closest isotropic elasticity tensor to the orthotropic tensor given in Table 4, several different measures can be applied (e.g. Gazis et al., 1963; Bos and Slawinski, 2015). We perform a least squares inversion which leads to the following analytical solutions for the equivalent 1-D isotropic $\mu$ and $\mu_{c}$ values

Table 4

Micropolar constants given in Eringen's book (Eringen, 1999) for potassium nitrate $\left(\mathrm{KNO}_{3}\right)$.

\begin{tabular}{|c|c|c|c|c|c|c|c|c|c|}
\hline$\rho\left[\frac{\mathrm{kg}}{\mathrm{m}^{3}}\right]$ & $\eta\left[\frac{\mathrm{kg}}{\mathrm{m}}\right]$ & $\mathbb{C}_{1111}\left[\frac{\mathrm{N}}{\mathrm{m}^{2}}\right]$ & $\mathbb{C}_{1221}\left[\frac{\mathrm{N}}{\mathrm{m}^{2}}\right]$ & $\mathbb{C}_{2121}\left[\frac{\mathrm{N}}{\mathrm{m}^{2}}\right]$ & $\mathbb{C}_{1212}\left[\frac{\mathrm{N}}{\mathrm{m}^{2}}\right]$ & $\mathbb{C}_{2112}\left[\frac{\mathrm{N}}{\mathrm{m}^{2}}\right]$ & $\mu \llbracket_{c}^{2}[\mathrm{~N}]$ & $v_{p}\left[\frac{\mathrm{m}}{\mathrm{s}}\right]$ & $v_{s}\left[\frac{\mathrm{m}}{\mathrm{s}}\right]$ \\
\hline 340 & $2.15 \times 10^{-18}$ & $1.31 \times 10^{10}$ & $0.72 \times 10^{10}$ & $0.76 \times 10^{10}$ & $0.68 \times 10^{10}$ & $0.71 \times 10^{10}$ & 0 & 6207.206 & 4472.136 \\
\hline
\end{tabular}




$$
\begin{aligned}
\mu & =\frac{\mathbb{C}_{1221}+\mathbb{C}_{1212}+\mathbb{C}_{2121}+\mathbb{C}_{2112}}{4} \\
& =7.175 \mathrm{GPa} \quad \text { and } \mu_{c} \\
& =\frac{\mathbb{C}_{1212}+\mathbb{C}_{2121}-\mathbb{C}_{1221}-\mathbb{C}_{2112}}{4}=0.025 \mathrm{GPa} .
\end{aligned}
$$

The Cosserat ratio is given by $\mathrm{r}_{\mu_{c}, \mu}=\mu_{c} / \mu=0.00349$.

\section{Appendix B. Finite-difference discretization}

The finite-difference (FD) scheme considered in this study is a simple conventional second-order scheme in time and space $\left(\mathscr{O}\left(\Delta t^{2}, \Delta x^{2}\right)\right)$, which may be readily written from the micropolar equations of motion (2.2) as follows

$$
\begin{aligned}
\rho \frac{u_{x}^{t+\Delta t}-2 u_{x}^{t}+u_{x}^{t-\Delta t}}{\Delta t^{2}}= & \left(\mu+\mu_{c}\right) \frac{u_{x+\Delta x}^{t}-2 u_{x}^{t}+u_{x-\Delta x}^{t}}{\Delta x^{2}} \\
& -2 \mu_{c} \frac{\vartheta_{x+\Delta x}^{t}-\vartheta_{x-\Delta x}^{t}}{2 \Delta x}+f\left(x_{s}, t\right)+\mathcal{O}\left(\Delta t^{2}, \Delta x^{2}\right),
\end{aligned}
$$$$
\eta \frac{\vartheta_{x}^{t+\Delta t}-2 \vartheta_{x}^{t}+\vartheta_{x}^{t-\Delta t}}{\Delta t^{2}}=\mu L_{c}^{2} \frac{\vartheta_{x+\Delta x}^{t}-2 \vartheta_{x}^{t}+\vartheta_{x-\Delta x}^{t}}{\Delta x^{2}}+2 \mu_{c} \frac{u_{x+\Delta x}^{t}-u_{x-\Delta x}^{t}}{2 \Delta x}
$$$$
-4 \mu_{c} \vartheta_{x}^{t}+\mathcal{O}\left(\Delta t^{2}, \Delta x^{2}\right),
$$

where $f$ is the source time functions applied over the displacement $u$ field. The Ricker source used in the numerical experiments is given by the following expression

$f\left(x_{s}, t\right)=\delta\left(x-x_{s}\right)\left(1-2 f_{0}^{2}\left(t-t_{0}\right)^{2}\right) e^{-f_{0}^{2}\left(t-t_{0}\right)^{2}}$,

where $\delta$ is the Dirac delta function, $t_{0}$ the time delay and $f_{0}$ the dominant frequency.

\section{References}

Abreu, R., Kamm, J., Reiß, A.-S., 2016. Micropolar modelling of rotational waves in seismology. Geophys. J. Int. Submitted for publication.

Askar, A., 1972. Molecular crystals and the polar theories of the continua. Experimental values of material coefficients for $\mathrm{KNO}_{3}$. Int. J. Eng. Sci. 10 (3), 293-300.

Bercovici, D., Ricard, Y., 2012. Mechanisms for the generation of plate tectonics by two-phase grain-damage and pinning. Phys. Earth Planet. Inter. 202-203, 2755.

Bercovici, D., Ricard, Y., 2014. Plate tectonics, damage and inheritance. Nature 508 (7497), 513-516.

Bernauer, M., Fichtner, A., Igel, H., 2009. Inferring earth structure from combined measurements of rotational and translational ground motions. Geophysics 74 (6), WCD41-WCD47.

Borkar, H., Singh, V.N., Singh, B.P., Tomar, M., Gupta, V., Kumar, A., 2014. Room temperature lead-free relaxor-antiferroelectric electroceramics for energy storage applications. RSC Adv. 4, 22840-22847.

Bos, L., Slawinski, M., 2015. 2-norm effective isotropic Hookean solids. J. Elast. 120 (1), $1-22$.

Bukowinski, M., Chizmeshya, A., Wolf, G., Zhang, H., 1996. Advances in electron-gas potential models: applications to some candidate lower mantle minerals. Mol. Eng. 6 (1-2), 81-112.

Chen, Y., Lee, J.D., Eskandarian, A., 2004. Atomistic viewpoint of the applicability of microcontinuum theories. Int. J. Solids Struct. 41 (8), 2085-2097.

Chopelas, A., 1996. Thermal expansivity of lower mantle phases $\mathrm{MgO}$ and $\mathrm{MgSiO}_{3}$ perovskite at high pressure derived from vibrational spectroscopy. Phys. Earth Planet. Inter. 98 (1), 3-15.

Cordier, P., Demouchy, S., Beausir, B., Taupin, V., Barou, F., Fressengeas, C., 2014. Disclinations provide the missing mechanism for deforming olivine-rich rocks in the mantle. Nature 507 (7490), 51-56.

Cosserat, E., Cosserat, F., 1909. Théorie des Corps Déformables. Librairie Scientifique, A. Hermann et Fils, (english translation by D. Delphenich 2007, pdf available at https://www.uni-due.de/hm0014/Cosseratles/Cosserat09eng.pdf), reprint 2009 by Hermann Librairie Scientifique, ISBN 978270566920 1, Paris.

Cowley, R., 1964. Lattice dynamics and phase transitions of strontium titanate. Phys. Rev. 134 (4A), A981.

Durand, S., Matas, J., Ford, S., Ricard, Y., Romanowicz, B., Montagner, J.-P., 2013. Insights from ScS-S measurements on deep mantle attenuation. Earth Planet. Sci. Lett. 374, 101-110.

Durben, D.J., Wolf, G.H., 1992. High-temperature behavior of metastable $\mathrm{MgSiO}_{3}$ perovskite: a Raman spectroscopic study. Am. Mineral. 77 (7-8), 890-893.

Durek, J.J., Ekström, G., 1996. A radial model of anelasticity consistent with longperiod surface-wave attenuation. Bull. Seismol. Soc. Am. 86 (1A), 144-158.
Dziewonski, A.M., Anderson, D.L., 1981. Preliminary reference Earth model. Phys. Earth Planet. Inter. 25 (4), 297-356.

Eringen, C., 1999. Microcontinuum Field Theories I: Foundations and Solids. Springer.

Eringen, C., Kafadar, C., 1976. Polar Field Theories, Continuum Physics, vol. IV. Academic Press, pp. 1-75.

Ferreira, A.M.G., Igel, H., 2009. Rotational motions of seismic surface waves in a laterally heterogeneous Earth. Bull. Seismol. Soc. Am. 99 (2B), 1429-1436.

Forest, S., Sievert, R., 2003. Elastoviscoplastic constitutive frameworks for generalized continua. Acta Mech. 160 (1), 71-111.

Gade, M., Raghukanth, S., 2016. Seismic response of reduced micropolar elastic halfspace. J. Seismolog. 20 (3), 787-801.

Garnero, E.J., Helmberger, D.V., 1993. Travel times of S and SKS: implications for three-dimensional lower mantle structure beneath the central Pacific. J. Geophys. Res.: Solid Earth 98 (B5), 8225-8241.

Gazis, D.C., Tadjbakhsh, I., Toupin, R.A., 1963. The elastic tensor of given symmetry nearest to an anisotropic elastic tensor. Acta Crystallogr. A 16 (9), 917-922.

Ghose, S., Choudhury, N., Chaplot, S.L., Pal Chowdhury, C., Sharma, S.K., 1994. Lattice dynamics and Raman spectroscopy of protoenstatite $\mathrm{Mg}_{2} \mathrm{Si}_{2} \mathrm{O}_{6}$. Phys. Chem. Miner. 20 (7), 469-477.

Grekova, E., 2012a. Linear reduced cosserat medium with spherical tensor of inertia where rotations are not observed in experiment. Mech. Solids 47 (5), 538-543.

Grekova, E., 2012b. Nonlinear isotropic elastic reduced Cosserat continuum as a possible model for geomedium and geomaterials. Spherical prestressed state in the semilinear material. J. Seismolog. 16 (4), 695-707.

Grekova, E., 2016. Plane waves in the linear elastic reduced Cosserat medium with a finite axially symmetric coupling between volumetric and rotational strains. Math. Mech. Solids 21 (1), 73-93.

Grekova, E., Kulesh, M., Herman, G., 2009. Waves in linear elastic media with microrotations, Part 2: isotropic reduced Cosserat model. Bull. Seismol. Soc. Am. 99 (2B), 1423-1428.

Hemley, R., Jackson, M., Gordon, R., 1987. Theoretical study of the structure, lattice dynamics, and equations of state of perovskite-type $\mathrm{MgSiO}_{3}$ and $\mathrm{CaSiO}_{3}$. Phys Chem. Miner. 14 (1), 2-12.

Heywang, W., Lubitz, K., Wersing, W., 2008. Piezoelectricity: Evolution and Future of a Technology, vol. 114. Springer.

Hier-Majumder, S., 2014. Melt redistribution by pulsed compaction within UltraLow Velocity Zones. Phys. Earth Planet. Inter. 229, 134-143.

Hirai, S., Kojima, Y., Ohfuji, H., Nishiyama, N., Irifune, T., Klemme, S., Bromiley, G., Attfield, J.P., 2011. High-pressure Raman studies and heat capacity measurements on the $\mathrm{MgSiO}_{3}$ analogue $\mathrm{CaIr}_{0.5} \mathrm{Pt}_{0.5} \mathrm{O}_{3}$. Phys. Chem. Miner. 38 (8), 631-637.

Hiraiwa, M., Abi Ghanem, M., Wallen, S.P., Khanolkar, A., Maznev, A.A., Boechler, N. 2016. Complex contact-based dynamics of microsphere monolayers revealed by resonant attenuation of surface acoustic waves. Phys. Rev. Lett. 116 (19) 198001.

Hwang, Y.K. Ritsema, J., 2011. Radial $Q_{\mu}$ structure of the lower mantle from teleseismic body-wave spectra. Earth Planet. Sci. Lett. 303 (3-4), 369-375.

Igel, H., Schreiber, U., Flaws, A., Schuberth, B., Velikoseltsev, A., Cochard, A., 2005 Rotational motions induced by the M8.1 Tokachi-oki earthquake, September 25, 2003. Geophys. Res. Lett. 32, L08309.

Igel, H., Cochard, A., Wassermann, J., Flaws, A., Schreiber, U., Velikoseltsev, A., Dinh, N.P., 2007. Broad-band observations of earthquake-induced rotational ground motions. Geophys. J. Int. 168 (1), 182-197.

Jeong, J., Neff, P., 2010. Existence, uniqueness and stability in linear Cosserat elasticity for weakest curvature conditions. Math. Mech. Solids 15 (1), 78-95.

Karki, B., Wentzcovitch, R., De Gironcoli, S., Baroni, S., 2000. Ab initio lattice dynamics of $\mathrm{MgSiO}_{3}$ perovskite at high pressure. Physical Review B 62 (22), 14750.

Kissel, C., Laj, C., 2012. Paleomagnetic Rotations and Continental Deformation, vol. 254. Springer.

Krupanidhi, S., 2003. Relaxor type perovskites: primary candidates of nano-polar regions. J. Chem. Sci. 115 (5-6), 775-788.

Kulesh, M., Grekova, E., Shardakov, I., 2009. The problem of surface wave propagation in a reduced Cosserat medium. Acoust. Phys. 55 (2), 218-226.

Lawrence, J.F., Wysession, M.E., 2006. QLM9: A new radial quality factor $\left(\mathbf{Q}_{\mu}\right)$ mode for the lower mantle. Earth Planet. Sci. Lett. 241 (3-4), 962-971.

Lee, W.H.K., Huang, B.-S., Langston, C.A., Lin, C.-J., Liu, C.-C., Shin, T.-C., Teng, T.-L. $\mathrm{Wu}, \mathrm{C} .-\mathrm{F} ., 2009$. Review: progress in rotational ground-motion observations from explosions and local earthquakes in Taiwan. Bull. Seismol. Soc. Am. 99 (2B), 958-967.

Liu, J., Li, J., Hrubiak, R., Smith, J.S., 2016. Origins of ultralow velocity zones through slab-derived metallic melt. Proc. Nat. Acad. Sci. 113 (20), 5547-5551.

Luyendyk, B.P., Kamerling, M.J., Terres, R., 1980. Geometric model for Neogene crustal rotations in southern California. GSA Bulletin 91 (4), 211-217.

Madeo, A., Barbagallo, G., d'Agostino, M.V., Neff, P. 2016a. First evidence of nonlocality in real band-gap metamaterials: determining parameters in the relaxed micromorphic model. Preprint arXiv:1603.02258 - to appear in Proceedings of the Royal Society A (London).

Madeo, A., Neff, P., d'Agostino, M.V., Barbagallo, G., 2016b. Complete band gaps including non-local effects occur only in the relaxed micromorphic model. Preprint arXiv:1602.04315.

Madeo, A., Neff, P., Ghiba, I.-D., Rosi, G. 2016c. Reection and transmission of elastic waves at interfaces embedded in non-local band-gap metamaterials: a comprehensive study via the relaxed micromorphic model. Preprint arXiv:1602.05218 - to appear in Journal of the Mechanics and Physics of Solids. 
Merkel, A., Luding, S., 2017. Enhanced micropolar model for wave propagation in ordered granular materials. Int. J. Solids Struct. 106, 91-105.

Merkel, A., Tournat, V., Gusev, V., 2011. Experimental evidence of rotational elastic waves in granular phononic crystals. Phys. Rev. Lett. 107 (22), 225502.

Mindlin, R., 1964. Microstructure in linear elasticity. Arch. Ration. Mech. Anal. 16, $51-78$.

Mouraille, O., Mulder, W.A., Luding, S., 2006. Sound wave acceleration in granular materials. J. Stat. Mech: Theory Exp. 2006 (07), P07023.

Murakami, M., Ohishi, Y., Hirao, N., Hirose, K., 2012. A perovskitic lower mantle inferred from high-pressure, high-temperature sound velocity data. Nature 485 (7396), 90-94.

Nagahama, H., Teisseyre, R., 2000a. Micromorphic continuum and fractal fracturing in the lithosphere. Pure Appl. Geophys. 157 (4), 559-574.

Nagahama, H., Teisseyre, R., 2000b. Micromorphic continuum and fractal properties of faults and earthquakes. Earthquake Thermodyn. Phase Transformations Earth's Interior, 425-440.

Neff, P., Jeong, J., 2009. A new paradigm: the linear isotropic Cosserat model with conformally invariant curvature energy. Zeitschrift für Angewandte Mathematik und Mechanik 89 (2), 107-122.

Neff, P., Jeong, J., Fischle, A., 2010a. Stable identification of linear isotropic Cosserat parameters: bounded stiffness in bending and torsion implies conformal invariance of curvature. Acta Mech. 211 (3-4), 237-249.

Neff, P., Jeong, J., Münch, I., Ramézani, H., 2010b. Linear Cosserat elasticity, conformal curvature and bounded stiffness. Mechanics of Generalized Continua. Springer, pp. 55-63.

Nowacki, W., 1986. Theory of Micropolar Elasticity. Pergamon Press.

Oganov, A.R., Ono, S., 2004. Theoretical and experimental evidence for a postperovskite phase of $\mathrm{MgSiO}_{3}$ in Earth's D layer. Nature 430 (6998), 445-448.

Parlinski, K., Kawazoe, Y., 2000. Ab initio study of phonons and structural stabilities of the perovskite-type MgSiO3. Eur. Phys. J. B-Condensed Matter Complex Syst. 16 (1), 49-58.

Peláiz-Barranco, A., González-Carmenate, I., Calderón-Pinar, F., 2005. Relaxor behaviour in PZN-PT-BT ferroelectric ceramics. Solid State Commun. 134 (8), 519-522.

Pouget, J., Aşkar, A., Maugin, G., 1986a. Lattice model for elastic ferroelectric crystals: continuum approximation. Phys. Rev. B 33 (9), 6320.

Pouget, J., Așkar, A., Maugin, G., 1986b. Lattice model for elastic ferroelectric crystals: microscopic approach. Phys. Rev. B 33 (9), 6304.

Randall, C.A., Bhalla, A.S., Shrout, T.R., Cross, L.E., 1990. Relationship between B-site order and properties in $\mathrm{Pb}(\mathrm{B} 0 \mathrm{~B} 00) 03$ perovskites. Ferroelectrics Lett. Sect. 11 (5), 103-106.

Romanowicz, B., Mitchell, B., 2007. 1.21 - Deep Earth Structure - Q of the Earth from Crust to Core. In: Schubert, G. (Ed.), Treatise on Geophysics. Elsevier, Amsterdam, pp. 731-774.

Romeo, M., 2015. Acoustic waves in micropolar elastic ferroelectrics. Mech. Res. Commun. 63, 33-38.

Schreiber, U., Stedman, G., Igel, H., Flaws, A., 2006. Ring laser gyroscopes as rotation sensors for seismic wave studies. In: Teisseyre, R., Majewski, E., Takeo, M. (Eds.), Earthquake Source Asymmetry, Structural Media and Rotation Effects, pp. 377390.

Schwartz, L.M., Johnson, D.L., Feng, S., 1984. Vibrational modes in granular materials. Phys. Rev. Lett. 52 (10), 831-834.

Shim, S.-H., Kubo, A., Duffy, T.S., 2007. Raman spectroscopy of perovskite and postperovskite phases of $\mathrm{MgGeO}_{3}$ to 123 GPa. Earth Planet. Sci. Lett. 260 (1), 166 178.

Smiga, W., Garbarz-Glos, B., 2012. Studies of the influence of uniaxial pressure on the electric behaviour of Li0.015Na0.985NbO3 ceramics. Ukrainian J. Phys. Opt. $13,27$.
Solomatov, V.S., Reese, C.C., 2008. Grain size variations in the Earth's mantle and the evolution of primordial chemical heterogeneities. J. Geophys. Res.: Solid Earth 113 (B7), B07408. B07408.

Suryanto, W., Igel, H., Wassermann, J., Cochard, A., Schuberth, B., Vollmer, D., Scherbaum, F., Schreiber, U., Velikoseltsev, A., 2006. First comparison of arrayderived rotational ground motions with direct ring laser measurements. Bull. Seismol. Soc. Am. 96 (6), 2059-2071.

Takei, Y., Fujisawa, K., McCarthy, C., 2011. Experimental study of attenuation and dispersion over a broad frequency range: 1. The apparatus. J. Geophys. Res.: Solid Earth 116 (B9), B09204. B09204.

Teisseyre, R., 1973. Earthquake processes in a micromorphic continuum. Pure Appl. Geophys. 102 (1), 15-28.

Teisseyre, R., 2008a. Friction and fracture induced anisotropy: asymmetric stresses. In: Teisseyre, R., Nagahama, H., Majewski, E. (Eds.), Physics of Asymmetric Continuum: Extreme and Fracture Processes. Springer, pp. 163-169.

Teisseyre, R., 2011. Why rotation seismology: confrontation between classic and asymmetric theories. Bull. Seismol. Soc. Am. 101 (4), 1683-1691.

Teisseyre, R., Takeo, M., Majewski, E., 2006. Earthquake Source Asymmetry, Structural Media and Rotation Effects. Springer.

Teisseyre, R., Gorski, M., Teisseyre, K., 2008b. Fracture processes: spin and twist-shear coincidence. In: Teisseyre, R., Nagahama, H., Majewski, E. (Eds.), Physics of Asymmetric Continuum: Extreme and Fracture Processes. Springer, pp. 111-122.

Thorne, M.S., Garnero, E.J., 2004. Inferences on ultralow-velocity zone structure from a global analysis of SPdKS waves. J. Geophys. Res.: Solid Earth 109 (B08301), 1-22.

Thorne, M.S., Zhang, Y., Ritsema, J., 2013, Evaluation of 1-D and 3-D seismic models of the Pacific lower mantle with S, SKS, and SKKS traveltimes and amplitudes. J. Geophys. Res.: Solid Earth 118 (3), 985-995.

Twiss, R.J., 2009. An asymmetric micropolar moment tensor derived from a discrete-block model for a rotating granular substructure. Bull. Seismol. Soc. Am. 99 (2B), 1103-1131.

Twiss, R.J., Souter, B.J., Unruh, J.R., 1993. The effect of block rotations on the global seismic moment tensor and the patterns of seismic P and T axes. J. Geophys. Res.: Solid Earth 98 (B1), 645-674.

Twiss, R.J., Souter, B.J., Unruh, J.R., 1993. The effect of block rotations on the global seismic moment tensor and the patterns of seismic P and T axes. J. Geophys. Res.: Solid Earth 98 (B1), 645-674.

Wassermann, J., Lehndorfer, S., Igel, H., Schreiber, U., 2009. Performance test of a commercial rotational motions sensor. Bull. Seismol. Soc. Am. 99 (2B), 14491456.

Wells, R.E., Heller, P.L., 1988. The relative contribution of accretion, shear, and extension to Cenozoic tectonic rotation in the Pacific Northwest. GSA Bull. 100 (3), 325-338.

Widmer, R., Masters, G., Gilbert, F., 1991. Spherically symmetric attenuation within the Earth from normal mode data. Geophys. J. Int. 104 (3), 541.

Williams, Q., Jeanloz, R., McMillan, P., 1987. Vibrational spectrum of MgSiO3 perovskite: zero-pressure Raman and mid-infrared spectra to $27 \mathrm{GPa}$. J. Geophys. Res.: Solid Earth 92 (B8).

Yin, J., Nigbor, R.L., Chen, Q., Steidl, J., 2016. Engineering analysis of measured rotational ground motions at \{GVDA\}. Soil Dyn. Earthquake Eng. 87, 125-137.

Zaroli, C., Debayle, E., Sambridge, M., 2010. Frequency-dependent effects on global S-wave traveltimes: wavefront-healing, scattering and attenuation. Geophys. J. Int. 182 (2), 1025-1042.

Zheng, Q.-S., Spencer, A., 1993. On the canonical representations for Kronecker powers of orthogonal tensors with application to material symmetry problems. Int. J. Eng. Sci. 31 (4), 617-635. 